Open Access

\title{
Determination of Target Values of Engineering Characteristics in QFD Using Uncertain Programming
}

\author{
Yunwen Miao ${ }^{1}$, Yuanyuan Liu ${ }^{1 *}$ and Yizeng Chen ${ }^{2}$
}

\author{
${ }^{*}$ Correspondence: \\ liuyuanyuan@shu.edu.cn \\ 1 School of Management, Shanghai \\ University, 99 Shangda Road, \\ 200444 Shanghai, China \\ Full list of author information is \\ available at the end of the article
}

\begin{abstract}
Quality function deployment (QFD) is a new product development tool remarked with interpreting customer requirements into engineering characteristics of the design process. On account of the inherent imprecise and uncertain elements in the weights of customer requirements, the relationships between customer requirements and engineering characteristics, and the correlations among engineering characteristics, uncertain variables are preferred to be applied in this paper. By taking advantage of expected value modelling to determine the target values of engineering characteristics in handling different practical design scenarios, two uncertain programming models are proposed for optimizing the QFD process in an uncertain environment. Subsequently, the proposed uncertain models are implemented in a motor car design for quality development.
\end{abstract}

Keywords: Quality function deployment, Engineering characteristic, Uncertain variable, Expected value modelling

\section{Introduction}

Nowadays, global economy has raised fierce international market competition and rapid technological change, especially in traditional tangible products made by manufacturing enterprises. For the sake of occupying sales and profits in larger market and long-term development, more and more economical products with high quality are designed and generated to cater diverse customer perceptions and expectations. As far as the continuous development of an existing product is concerned, the use of quality function development (QFD) has gained extensive global support. Originated in Japan in the late 1960s [1], QFD was known as a customer-driven product design methodology, which is meant to promote the quality of products. It is a systematic method contributing to translating various customer requirements (CRs) into several engineering characteristics (ECs) of the product for achieving higher customer satisfaction. So far, QFD has been applied in many other fields, including supply chain management [2, 3], investment [4], product selection and assignment [5], etc.

The core concept of QFD is to utilize four sets of matrices integrated in a diagram that resembles a house, namely, the House of Quality (HoQ) [6]. As demonstrated in Fig. 1, CRs can be considered as whats, specific descriptions and the relative importance of

(C) 2015 Miao et al. Open Access This article is distributed under the terms of the Creative Commons Attribution 4.0 International License (http://creativecommons.org/licenses/by/4.0/), which permits unrestricted use, distribution, and reproduction in any medium, provided you give appropriate credit to the original author(s) and the source, provide a link to the Creative Commons license, and indicate if changes were made. 


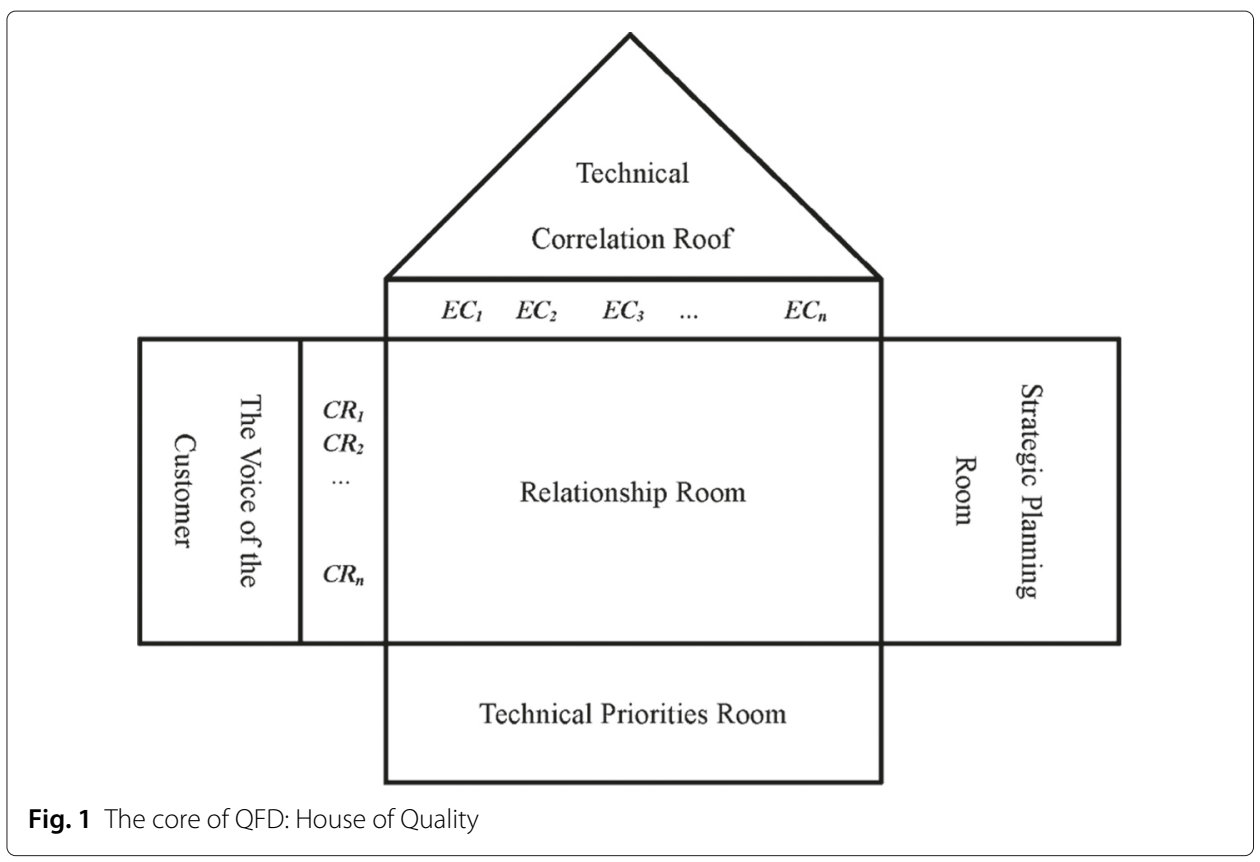

which will be listed in the matrix on the left wall of HoQ, while ECs are on behalf of hows of a product to meet these demands from customers. The body of HoQ is the relationship matrix of the whats and hows, and the roof is the correlation matrix that illustrates the dependence among the hows. Besides, the matrix of target values of ECs on the floor provides the quantitative technical specifications for ECs required to satisfy CRs.

The product development process based on QFD is to determine a set of $x_{1}, x_{2}, \cdots, x_{n}$ for ECs of the new/improved product to match or exceed the degree of overall customer satisfaction of all competitors in the target market with limited organizational resources. It is a complex decision process with multiple variables, requiring trade-off and optimizing all kinds of conflicts contained in HoQ. As an important branch of QFD research, more and more systematic and rational programmings with different considerations to determine the target values of ECs have achieved flourishing advances in the last few decades. Generally, a traditional and classic programming in QFD planning is designed to achieve maximum overall customer satisfaction in the constraints of two functional relationships including levels of attainment between CRs and ECs and that among ECs, and other constraints of resources including cost, resource, technology, etc.

In order to formulate the programming, it is critical to first determine the relative importance of CRs and the functional relationships. According to the previous literature, they were either confirmed by subjective assessments and judgments expressed as crisp, random or fuzzy variables [7-10], or by the frequent application of the fuzzy linear and non-linear regressions methods [11-14]. Even though the latter one seems more objective, practically speaking, it is much less feasible due to sparse data collection.

It can be seen that, among them, fuzzy modelling approaches were popular since they have applied fuzziness based on the fuzzy set theory [15] to define the imprecise elements in HoQ. Therefore, the objective function and constraints of different programmings can be incorporated with fuzzy parameters to get better results close to reality. On this basis, numerous studies have been conducted on how to obtain a set of target values of ECs. 
Chen et al. proposed a fuzzy expected value modelling approach for target setting, which simultaneously took maximizing the overall customer satisfaction and minimizing the design cost into consideration [16]. Sener and Karsak developed some fuzzy mathematical programming models combining the functional relationships obtained from a fuzzy regression based on non-linear programming and an integrated fuzzy linear regression and fuzzy multiple objective programming approach to determining target values of ECs $[17,18]$. Zhong et al. proposed a fuzzy chance-constrained programming model with the objective of minimizing the fuzzy expected cost and the chance constraint of overall customer satisfaction [19].

Until now, most of the variables or parameters applied in QFD process were either crisp values or fuzzy ones. However, it is usually not appropriate enough because both the probability theory and the fuzzy set theory may sometimes lead to counterintuitive results [20]. In this paper, we put forward a new method based on the uncertainty theory proposed by Liu [21] and redefined in Liu [22]. Similarly to fuzzy optimization models, the uncertain variables involved like the relative importance of CRs, the uncertain relationship between CRs and ECs, the uncertain correlations among ECs and the variable cost to fulfill one unit of ECs, will be predefined by experts in a vague way using uncertain variables rather than crisp values. So as to effectively determine the target values of ECs in handling practical design scenarios, two uncertain programming models using expected value modelling (EVM) are generated under the objectives of maximizing the overall customer satisfaction and minimizing the total design cost, respectively.

The rest of the article is organized as follows. In Section Preliminaries, some preliminaries of uncertain variable, uncertainty distribution, and uncertain programming are described. In Section Uncertainty theory, two uncertain programming models using EVM for QFD planning in an uncertain environment are proposed to determine the target values of ECs. Finally, Section Uncertain Expected Value Modelling for QFD Planning illustrates a numerical example of a motor car design, which is presented to demonstrate the performance of the proposed approach.

\section{Preliminaries}

Uncertainty theory is an efficient mathematical system to deal with indeterminacy, which plays an crucial role to measure expert statistics and subjective estimations. In this section, some basic knowledge of uncertainty theory is introduced for describing the approaches of EVM applied in the uncertain programming method. The reader may refer to Liu [20-22] for more details.

\section{Uncertainty theory}

Definition 1. (Liu [21]) Let $\Gamma$ be a nonempty set, and $\mathcal{L}$ a $\sigma$-algebra over $\Gamma$. The set function $\mathcal{M}: \mathcal{L} \rightarrow[0,1]$ is called an uncertain measure if it satisfies normality, duality and subadditivity axioms as follows:

(i) $\mathcal{M}\{\Gamma\}=1$ for the universal set $\Gamma$;

(ii) $\mathcal{M}\{\Lambda\}+\mathcal{M}\left\{\Lambda^{c}\right\}=1$ for any event $\Lambda$;

(iii) For every countable sequence of events $\Lambda_{1}, \Lambda_{2}, \cdots$, we have

$$
\mathcal{M}\left\{\bigcup_{i=1}^{\infty} \Lambda_{i}\right\} \leq \sum_{i=1}^{\infty} \mathcal{M}\left\{\Lambda_{i}\right\} .
$$


In addition, in order to introduce the operational law, the product uncertain measure $\mathcal{M}$ on the product $\sigma$-algebra $\mathcal{L}$ is defined by the following product axiom according to Liu [23]:

(iv) Let $\left(\Gamma_{k}, \mathcal{L}_{k}, \mathcal{M}_{k}\right)$ be uncertainty spaces for $k=1,2, \ldots$. Then the product uncertain measure $\mathcal{M}$ is an uncertain measure satisfying

$$
\mathcal{M}\left\{\prod_{k=1}^{\infty} \Lambda_{k}\right\}=\bigwedge_{k=1}^{\infty} \mathcal{M}_{k}\left\{\Lambda_{k}\right\}
$$

where $\Lambda_{k}$ are arbitrarily chosen events from $\mathcal{L}_{k}$ for $k=1,2, \cdots$, respectively.

Based on the four axioms of uncertain measure given above, a formal definition of uncertain variable is presented as follows.

Definition 2. (Liu [21]) An uncertain variable is a measurable function $\xi$ from an uncertainty space $(\Gamma, \mathcal{L}, \mathcal{M})$ to the set of real numbers such that $\{\xi \in B\}$ is an event for any Borel set $B$.

To better describe uncertain variables, the concept of uncertainty distribution is adopted. In many cases, it is more sufficient to know the uncertainty distribution than the uncertain variable itself.

Definition 3. (Liu [21]) The uncertainty distribution $\Phi$ of an uncertain variable $\xi$ is defined by

$$
\Phi(x)=\mathcal{M}\{\xi \leq x\}
$$

for any real number $x$.

Furthermore, the regular uncertainty distribution is defined as follows, which is shown in Fig. 2.

Definition 4. (Liu [22]) An uncertainty distribution $\Phi(x)$ is said to be regular if it is a continuous and strictly increasing function with respect to $x$ at which $0<\Phi(x)<1$, and

$$
\lim _{x \rightarrow-\infty} \Phi(x)=0, \quad \lim _{x \rightarrow+\infty} \Phi(x)=1
$$

Suppose that a regular uncertainty distribution $\Phi(x)$ has an inverse function on the range of $x$ with $0<\Phi(x)<1$, and the inverse function $\Phi^{-1}(\alpha)$ exists on the open interval $(0,1)$ and is unique for each $\alpha \in(0,1)$, then the inverse function $\Phi^{-1}(\alpha)$ is called the inverse uncertainty distribution of an uncertain variable $\xi$, which is vital in operations of independent uncertain variables with regular uncertainty distributions.

In order to ensure that we can separately define uncertain variables on different uncertainty spaces, in 2009, Liu defined the independence of uncertain variables in the following mathematical forms. 


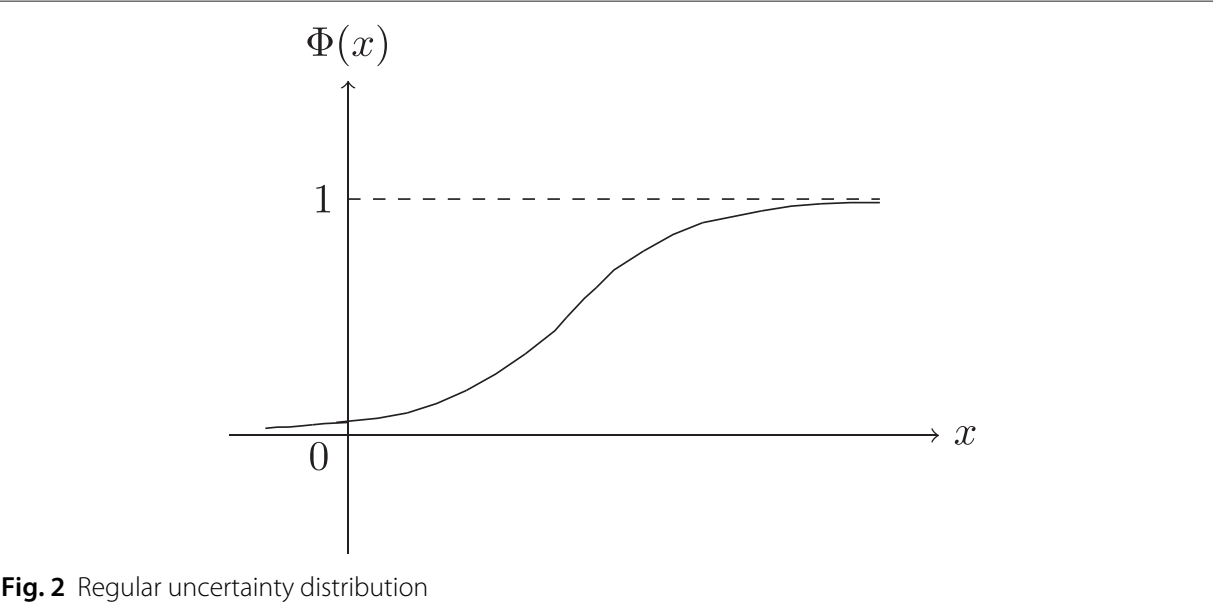

Fig. 2 Regular uncertainty distribution

Definition 5. (Liu [23]) The uncertain variables $\xi_{1}, \xi_{2}, \ldots, \xi_{n}$ are said to be independent if

$$
\mathcal{M}\left\{\bigcap_{i=1}^{n}\left\{\xi_{i} \in B_{i}\right\}\right\}=\bigwedge_{i=1}^{n} \mathcal{M}\left\{\xi_{i} \in B_{i}\right\}
$$

for any Borel sets $B_{1}, B_{2}, \ldots, B_{n}$ of real numbers.

Therefore, regarding strictly monotone functions of independent uncertain variables with regular uncertainty distributions, the operational law was given by Liu [22].

Theorem 1. (Liu [22]) Let $\xi_{1}, \xi_{2}, \cdots, \xi_{n}$ be independent uncertain variables with inverse uncertainty distributions $\Phi_{\xi_{1}}^{-1}, \Phi_{\xi_{2}}^{-1}, \ldots, \Phi_{\xi_{n}}^{-1}$, respectively. If the function $f\left(x_{1}, \cdots, x_{m}, x_{m+1}, \cdots, x_{n}\right)$ is strictly increasing with $x_{1}, \cdots, x_{m}$ and strictly decreasing with $x_{m+1}, \cdots, x_{n}$, then $\xi=f\left(\xi_{1}, \cdots, \xi_{m}, \xi_{m+1}, \cdots, \xi_{n}\right)$ is an uncertain variable with inverse uncertainty distribution

$$
\Psi_{\xi}^{-1}(\alpha)=f\left(\Phi_{\xi_{1}}^{-1}(\alpha), \cdots, \Phi_{\xi_{m}}^{-1}(\alpha), \Phi_{\xi_{m+1}}^{-1}(1-\alpha), \cdots, \Phi_{\xi_{n}}^{-1}(1-\alpha)\right) .
$$

With respect to uncertain measure, the expected value of an uncertain variable is the average value, which is able to be represented by the inverse uncertainty distribution as follows.

Definition 6. (Liu [21]) Let $\xi$ be an uncertain variable. Then the expected value of $\xi$ is defined by

$$
E[\xi]=\int_{0}^{+\infty} \mathcal{M}\{\xi \geq x\} \mathrm{d} x-\int_{-\infty}^{0} \mathcal{M}\{\xi \leq x\} \mathrm{d} x,
$$

provided that at least one of the two integrals is finite.

Theorem 2. (Liu [22]) Let $\xi$ be an uncertain variable with a regular uncertainty distribution. Then

$$
E[\xi]=\int_{0}^{1} \Phi^{-1}(\alpha) \mathrm{d} \alpha .
$$


Thus, referring to the operational law of strictly monotone function of independent uncertain variables, the expected value can be calculated as follows.

Theorem 3. (Liu and $\mathrm{Ha}$ [24]) Assume that $\xi_{1}, \xi_{2}, \cdots, \xi_{n}$ are independent uncertain variables with regular distributions $\Phi_{1}, \Phi_{2}, \cdots, \Phi_{n}$, respectively. If $f\left(x_{1}, x_{2}, \cdots, x_{n}\right)$ is strictly increasing with respect to $x_{1}, x_{2}, \cdots, x_{m}$ and strictly decreasing with respect to $x_{m+1}, x_{m+2}, \cdots, x_{n}$, then the uncertain variable $\xi=f\left(\xi_{1}, \xi_{2}, \cdots, \xi_{n}\right)$ has an expected value as

$$
E[\xi]=\int_{0}^{1} f\left(\Phi_{1}^{-1}(\alpha), \cdots, \Phi_{m}^{-1}(\alpha), \Phi_{m+1}^{-1}(1-\alpha), \cdots, \Phi_{n}^{-1}(1-\alpha)\right) \mathrm{d} \alpha
$$

\section{Uncertain Expected Value Modelling for QFD Planning}

QFD is a planning and problem-solving tool for product development, the core of which is House of Quality ( $\mathrm{HoQ})$ embedded with four matrices, i.e., relative importance matrix of CRs, relationship matrix between CRs and ECs, correlations matrix among ECs, and target value matrix of ECs.

According to information provided in $\mathrm{HoQ}$, the purpose of product planning process is usually to determine target values of ECs to maximize the overall customer satisfaction with limited organizational resources and technologies, or to minimize the design cost under a preferred acceptable overall customer satisfaction. In reality, we frequently lack observed data, and the estimated probability distribution may be far from the cumulative frequency $[20,25]$. In order to get over this difficulty, based on uncertainty theory, the uncertain programming method using EVM is proposed in this section to deal with QFD planning problem in an uncertain environment.

\section{Problem Notations and Explanations}

Assuming that $m$ CRs, $n$ ECs, and $p$ competitors are involved in a product design, the notations used in HoQ are summarized as follows,

- $\quad \mathrm{CR}_{i}$ : the $i$ th customer requirement, $i=1,2, \cdots, m$;

- $\quad \mathrm{EC}_{j}$ : the $j$ th engineering characteristic, $j=1,2, \cdots, n$;

- Comp $q$ : the $q$ th competitor, $q=1,2, \cdots, p$;

- $\quad R$ : the original uncertain relationship matrix between CRs and ECs, the element $r_{i j}$ of which denotes the uncertain relation measure between $\mathrm{CR}_{i}$ and $\mathrm{EC}_{j}$;

- $\quad P$ : the uncertain correlation matrix among ECs, the element $p_{k j}$ of which denotes the uncertain correlation measure between $\mathrm{EC}_{j}$ and $\mathrm{EC}_{k}$;

- $\quad R^{\prime}$ : the modified uncertain relationship matrix between CRs and ECs by accommodating the uncertain correlation matrix $P$, in which the element $r_{i j}^{\prime}$ denotes the modified uncertain relationship measure between $\mathrm{CR}_{i}$ and $\mathrm{EC}_{j}$;

- $\quad Y$ : the uncertain vector of customer perception of CRs, $Y=\left(y_{1}, y_{2}, \cdots, y_{m}\right)^{T}$, and $y_{i}$ is the customer perception of the satisfaction degree of $\mathrm{CR}_{i}, i=1,2, \cdots, m$;

- $\quad W$ : the uncertain relative importance vector of CRs, $W=\left(w_{1}, w_{2}, \cdots, w_{m}\right)^{T}$, among which $w_{i}$ is the uncertain relative importance of $\mathrm{CR}_{i}, i=1,2, \cdots, m$;

- $\quad X$ : the vector of level of attainment of ECs, $X=\left(x_{1}, x_{2}, \cdots, x_{n}\right)^{T}$, and $x_{j}$ is the level of attainment of $\mathrm{EC}_{j}, 0 \leq x_{j} \leq 1, j=1,2, \cdots, n$; 
- $\quad V$ : the importance vector of ECs, $V=\left(v_{1}, v_{2}, \cdots, v_{n}\right)^{T}$, in which $v_{j}$ is the uncertain importance of $\mathrm{EC}_{j}, j=1,2, \cdots, n$;

- $S_{q}$ : the overall customer satisfaction of the $q$ th competitor, $q=1,2, \cdots, p$;

- $\quad l_{j}$ : the target value of $\mathrm{EC}_{j}, j=1,2, \cdots, n$;

- $\quad C$ : the total product design cost;

- $\quad C_{F}$ : the fixed part of design cost;

- $\quad C_{V}$ : the variable part of design cost;

- $\quad C_{j}$ : the uncertain cost required for achieving $x_{j}, j=1,2, \cdots, n$;

- $\quad c_{j}$ : the uncertain cost required for improving each one unit of $\mathrm{EC}_{j}, j=1,2, \cdots, n$;

- $\quad B$ : the budget of product development.

As introduced above, owing to the subjective assessment of the relative importance $w_{i}$ in matrix $W$, the relationships $r_{i j}$ in matrix $R$, and correlations $p_{k j}$ in matrix $P$, it is more appropriate to use uncertain variables to describe these imprecise linguistic terms like "important/unimportant", "strong/weak", or "positive/negative". Here, we unify uncertain variables with a regular uncertainty distribution like

$$
\Phi(x)=\left\{\begin{array}{cl}
0, & \text { if } x<0 \\
x^{a}, & \text { if } 0 \leq x \leq 1 \\
1, & \text { if } x>1
\end{array}\right.
$$

As for the weight assessment of diverse CRs, before making improvements of a product, it is crucial for an enterprise to discover customers' needs in the first place. Most of all, investigation will be launched in the target market among users. The feedbacks collected after delivering questionnaires or conducting surveys will reflect what the customer wants about the product. Thereby, in Formula (8), the values of parameter $a$ in $\Phi(x)$ were assigned based on the relative importance of CRs, which is defined in interval $[0,1]$. If $a>1$, it is depicted in Fig. 3a. With the increasing of $x$, the value of $\mathcal{M}\left\{w_{i} \leq x\right\}$ increases faster and faster, which means the corresponding $w_{i}$ is relatively high and close to 1 . Under this circumstance, it can be interpreted as "important". If $a<1$, it is depicted in Fig. 3b. With the increasing of $x$, the value of $\mathcal{M}\left\{w_{i} \leq x\right\}$ increases slower and slower, which means the corresponding $w_{i}$ is relatively small and close to 0 . We can interpret this circumstance as "unimportant". And if $a=1$, it is illustrated as Fig. 3c, which represents "moderately important".

The relationships between CRs and ECs are generated in the relationship matrix $R$, the body of HoQ, in which, each vector can be denoted as $r_{i j}$. Generally, the relationships summit to a pile of pre-defined uncertain variables measured by experts. The forms of

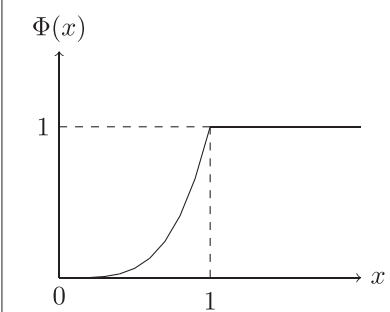

(a) Concave distribution

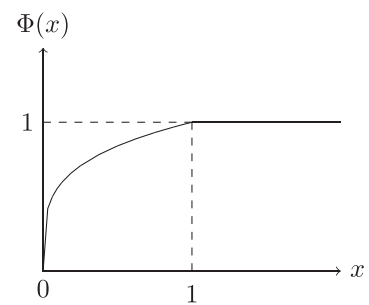

(b) Convex distribution

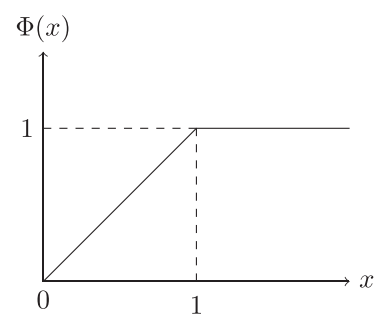

(c) Linear distribution

Fig. 3 An illustration of uncertain distribution of uncertain variables 
uncertain variables defined in Formula (8) can also be adopted to illustrate the strength of relationship, i.e., concave uncertainty distribution can explain the "strong" relationship between a certain CR and EC, while convex and linear ones represent "weak" and "medium", respectively.

Similarly, correlations among ECs can be represented as above uncertain variables defined by experts as well, which are illustrated in the correlation matrix $P$, the roof of $\mathrm{HoQ}$, and $P_{k j}$ denotes the correlation between $\mathrm{EC}_{k}$ and $\mathrm{EC}_{j}$. Concave and convex uncertainty distributions can reflect dependence like "positive" and "negative" among ECs, respectively. It is certain that, the $\mathrm{EC}$ is defined as the strongest dependence on itself in the construction of the correlation matrix [26], i.e., $p_{j j}$ is defined as the maximum degree in the correlation matrix.

\section{Normalizing the Target Values of ECs}

Generally, the target values of ECs in a product have been collected in different units, and usually, their ranges vary widely. In order to eliminate the influence of different measurements, referring to Chen [12], the target values of $\mathrm{EC}_{j}$ in a product, i.e., $l_{j}$, can be normalized into $x_{j}, j=1,2, \cdots, n$, according to the following transformation,

$$
x_{j}= \begin{cases}\frac{l_{j}^{\text {max }}-l_{j}}{l_{j}^{\text {max }}-l_{j}^{\text {min }}} & (S-\text { type }) \\ \frac{l_{j}-l_{j}^{\text {min }}}{l_{j}^{\text {max }}-l_{j}^{\text {min }}}, & (L-\text { type })\end{cases}
$$

where $l_{j}^{\max }$ and $l_{j}^{\text {min }}$ can be determined by the consideration of competition requirements and technology feasibility [27]. For $S$-type ECs, $l_{j}^{\max }$ is the maximum target value of $\mathrm{EC}_{j}$ that matches the performance of the main competitors, and $l_{j}^{\text {min }}$ is the minimized physical limit. Conversely, for L-type $\mathrm{ECs}, l_{j}^{\text {min }}$ is the minimum target value of $\mathrm{EC}_{j}$ that matches the performance of the main competitors, and $l_{j}^{\max }$ is the maximized physical limit. In this way, we can obtain the observation data matrix of ECs among $q$ companies, denoted by

$$
X=\left(x_{q j}\right)_{p \times n},
$$

where $0 \leq x_{q j} \leq 1$.

\section{Calculation of Overall Customer Satisfaction}

The overall customer satisfaction can be calculated through the integration of four matrices $W, R, P$, and $X$, which denote the relative importance of CRs, the relationships between CRs and ECs, the correlations among ECs and the target values of ECs, respectively.

Since it is crucial to establish trade-offs in analysis of the roof of HoQ, in essence, the correlation element $p_{k j}$ indicates the contribution to $\mathrm{EC}_{k}$ when $\mathrm{EC}_{j}$ is improved. Therefore, by accommodating the correlations among ECs [28], the original relationship matrix $R$ can be modified as

$$
R^{\prime}=R P,
$$

in which

$$
r_{i j}^{\prime}=\sum_{k=1}^{n} r_{i k} p_{k j}, i=1, \cdots, m, j=1, \cdots, n .
$$

The overall customer satisfaction of the product, $S$, can be considered as a mathematical aggregation of $y_{i}(i=1,2, \cdots, m)$, which implies the degree of satisfaction of $\mathrm{CR}_{i}$ in 
comparison among all the competitors. i.e., $S=f\left(y_{1}, y_{2}, \cdots, y_{m}\right)$, where $f\left(y_{1}, y_{2}, \cdots, y_{m}\right)$ is an aggregation function reflecting the customer's overall perceptions of the product. Thus, $S$ can be expressed as

$$
S=W^{T} Y=\sum_{i=1}^{m} w_{i} y_{i}
$$

It is the improvement of target values of ECs that will generate the increasing of the satisfaction degree of each CR [28], so we can obtain

$$
Y=R^{\prime} X,
$$

i.e.,

$$
y_{i}=\sum_{j=1}^{n} \sum_{k=1}^{n} r_{i k} p_{k j} x_{j}, i=1,2, \cdots, m .
$$

Thus, Formula (13) can be rewritten as

$$
S=W^{T} R^{\prime} X=V^{T} X=\sum_{j=1}^{n} v_{j} x_{j},
$$

where $V$ contains the vector of importance of ECs, and can be expressed as

$$
V=\left(W^{T} R^{\prime}\right)^{T} .
$$

Therefore, the importance of $\mathrm{EC}_{j}$ can be calculated by

$$
v_{j}=\sum_{i=1}^{m} \sum_{k=1}^{n} w_{i} r_{i k} p_{k j}, j=1,2, \cdots, n .
$$

Given a series values of ECs, the overall customer satisfaction can be obtained as

$$
S=\sum_{j=1}^{n}\left(\sum_{i=1}^{m} \sum_{k=1}^{n} w_{i} r_{i k} p_{k j}\right) x_{j} .
$$

\section{Formulation of Development Resources}

Multiple and diverse resources are required for the development of a product, which includes professional technical expertise, advanced equipment, tools, and other facilities. With respect to strategic planning, these resources can be integrated in financial terms. Owing to the uncertainties in the development process, such as incomplete understanding of the relationship between the CRs and the ECs, as well as the correlation among the $E C s$, thereby these financial terms can be expressed in uncertain variables. In general, the design cost, $C$, is combination of a fixed part denoted as $C_{F}$ and a variable part denoted as $C_{V}$, i.e.,

$$
C=C_{F}+C_{V}
$$

$C_{F}$ can be either a crisp value or an uncertain variable. In the case proposed in this paper, it is expressed as a crisp value, while the variable part of the design cost $C_{V}$ only depends on the levels of attainment of ECs, i.e., $x_{j}, j=1,2, \cdots, n$. Then, $C_{V}$ can be obtained by the sum of costs $C_{j}$ required for achieving the level of individual EC. For simplicity, suppose that $C_{j}$ is scaled linearly to the level of attainment $x_{j}$. Hence, we can obtain the variable cost with 


$$
C_{V}=\sum_{j=1}^{n} C_{j}=\sum_{j=1}^{n} c_{j} x_{j}, j=1,2, \cdots, n,
$$

where the cost coefficient $c_{j}$ denotes the cost needed when $\mathrm{EC}_{j}$ is fully improved, i.e., a cost $c_{j}$ will be required if one unit of attainment of the $\mathrm{EC}_{j}$ is fulfilled. Since the price of one unit material usually vibrates in an interval in the market, we adopt linear uncertain variables listed below to define $c_{j}$, which will be applied in the calculation later.

As shown in Fig. 4, the distribution of linear uncertain variable $\xi$ is formulated as

$$
\Phi(x)=\left\{\begin{array}{cl}
0, & \text { if } x \leq a \\
(x-a) /(b-a), & \text { if } a \leq x \leq b \\
1, & \text { if } x \geq b,
\end{array}\right.
$$

denoted by $\mathcal{L}(a, b)$, where $a$ and $b$ are real numbers with $a<b$. In this case, $a$ is the price of lower limit of one unit material in the market, and $b$ is the upper limit.

Hence, the total cost of development, $C$, can be expressed as

$$
C=C_{F}+C_{V}=C_{F}+\sum_{j=1}^{n} c_{j} x_{j}, j=1,2, \cdots, n .
$$

If the total cost of product development is constrained to a budget $B$, it can be represented as

$$
C_{F}+\sum_{j=1}^{n} c_{j} x_{j} \leq B
$$

\section{Uncertain Programming Using EVM}

Uncertain programming [29] has been adopted and improved to solving problems like network optimization in [30] and shortest path problem in [31] and so on. Recently, uncertain multi-objective programming and uncertain goal programming are generated by Liu and Chen [32] to apply in more research works. This paper used expected value modelling approach of uncertain programming, assuming that a set of $\left\{x_{1}, x_{2}, \cdots, x_{n}\right\}$ for ECs of a product are needed to be determined during the course of product planning, which aims to maximize the overall customer satisfaction under the organizational resources limits, it seems adoptable to generalize such an uncertain programming model for QFD as follows,

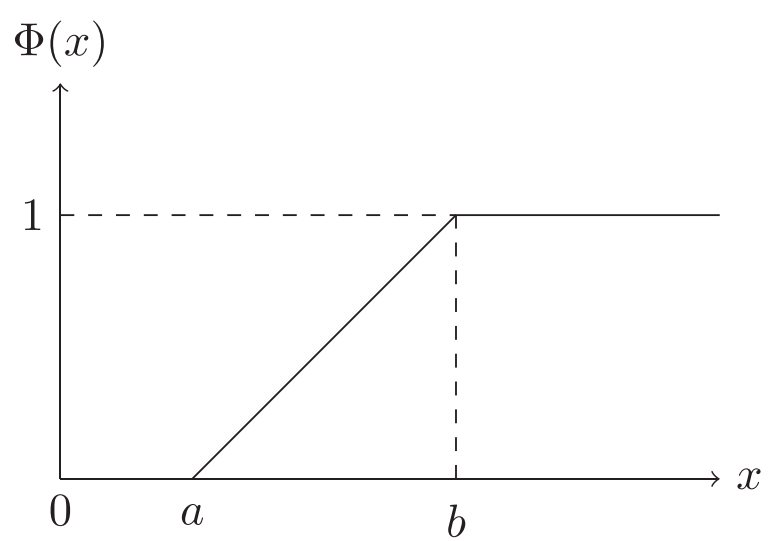

Fig. 4 Linear uncertain variable 


$$
\left\{\begin{array}{l}
\max S=\sum_{j=1}^{n} \sum_{i=1}^{m} \sum_{k=1}^{n} w_{i} r_{i k} p_{k j} x_{j} \\
\text { subject to: } \\
C_{F}+\sum_{j=1}^{n} c_{j} x_{j} \leq B \\
0 \leq x_{j} \leq 1, j=1,2, \cdots, n .
\end{array}\right.
$$

In the above objective functions and constraints, $w_{i}, r_{i k}, p_{k j}$, and $c_{j}$ are pre-defined uncertain variables mentioned in Section 3, the calculation of which will be described in more detail later. Notably, it seems quite appropriate and rational to utilize these uncertain variables in practical product design scenarios. However, it is hard for mathematical calculation since uncertain variables are not as straightforward as crisp ones.

For this reason, in order to build unambiguous uncertain programming model for QFD planning, two uncertain programming models using EVM are proposed in this section, in which the underlying philosophy is based on selecting the decision with the maximum expected returns.

Therefore, according to Section 3.3, the expected value of customer satisfaction $S$ in Formula (16) can be calculated as follows,

$$
E(S)=E\left(V^{T} X\right)=E\left(\sum_{j=1}^{n} v_{j} x_{j}\right)=\sum_{j=1}^{n} E\left(v_{j}\right) x_{j},
$$

where $E\left(v_{j}\right)$ is the expected value of importance of $\mathrm{EC}_{j}$ and can be obtained as

$$
E\left(v_{j}\right)=E\left(\sum_{i=1}^{m} \sum_{k=1}^{n} w_{i} r_{i k} p_{k j}\right)=\sum_{i=1}^{m} \sum_{k=1}^{n} E\left(w_{i} r_{i k} p_{k j}\right),
$$

in which, the uncertain variables $w_{i}, r_{i k}, p_{k j}$, are assumed to have regular uncertainty distributions. Assume the inverse uncertainty distributions of $w_{i}, r_{i k}$, and $p_{k j}$ are $\phi_{i}^{-1}(\alpha)$, $\Psi_{i k}^{-1}(\alpha)$ and $\Phi_{k j}^{-1}(\alpha)$, respectively. Hence, based on Theorem 3, $E\left(v_{j}\right)$ can be calculated as

$$
E\left(v_{j}\right)=\sum_{i=1}^{m} \sum_{k=1}^{n} E\left(w_{i} r_{i k} p_{k j}\right)=\sum_{i=1}^{m} \sum_{k=1}^{n}\left(\int_{0}^{1} \phi_{i}^{-1}(\alpha) \Psi_{i k}^{-1}(\alpha) \Phi_{k j}^{-1}(\alpha) \mathrm{d} \alpha\right) .
$$

The higher $E\left(v_{j}\right)$, the more important is $\mathrm{EC}_{j}$. If the organization resources are not considered, the EC with the highest expected value would be improved first. Furthermore, $E\left(v_{j}\right)$ can be scaled from 0 to 1 by

$$
\bar{E}\left(v_{j}\right)=\frac{E\left(v_{j}\right)}{\sum_{j=1}^{n} E\left(v_{j}\right)}
$$

where $\bar{E}\left(v_{j}\right)$ is the expected value of relative importance of ECs. Without loss of generality, by using $\bar{E}\left(v_{j}\right)$, the expcted value of the overall customer satisfaction can be normalized from 0 to 1 . Thus, Formula (26) can be rewritten as

$$
\bar{E}(S)=\sum_{j=1}^{n} \bar{E}\left(v_{j}\right) x_{j}
$$


where $\bar{E}(S)$ is the normalized expected value of the overall customer satisfaction.

Similar to the overall customer satisfaction, with respect to Section 3.4, the expected value of total cost $C$ can be calculated as

$$
E(C)=E\left(C_{F}+C_{V}\right)=C_{F}+E\left(C_{V}\right)=C_{F}+\sum_{j=1}^{n} E\left(c_{j}\right) x_{j},
$$

in which $c_{j}$ is the cost coefficient of $\mathrm{EC}_{j}$. Assume the inverse uncertainty distribution of linear uncertain variables $c_{j}$ is $\varphi_{j}^{-1}(\alpha)$, we can obtain the equivalent form of expected value of $C$ in Formula (31) as

$$
E(C)=C_{F}+\sum_{j=1}^{n} E\left(c_{j}\right) x_{j}=C_{F}+\sum_{j=1}^{n}\left(\int_{0}^{1} \varphi_{j}^{-1}(\alpha) \mathrm{d} \alpha\right) x_{j} .
$$

Owing to Theorem 2, the expected value of linear uncertain variables $c_{j}$ denoted by $\mathcal{L}\left(a_{j}, b_{j}\right)$ can be easily obtained as

$$
E\left(c_{j}\right)=\int_{0}^{1} \varphi_{j}^{-1}(\alpha) \mathrm{d} \alpha=\int_{0}^{1}\left((1-\alpha) a_{j}+\alpha b_{j}\right) \mathrm{d} \alpha=\frac{a_{j}+b_{j}}{2} .
$$

Therefore, when the design team wants to maximize the overall customer satisfaction under a limited budget, an uncertain programming model using EVM (UP-1) can be formulated as follows,

$$
\left\{\begin{array}{l}
\max \bar{E}(S)=\sum_{j=1}^{n} \bar{E}\left(v_{j}\right) x_{j} \\
\text { subject to: } \\
\quad C_{F}+\sum_{j=1}^{n} E\left(c_{j}\right) x_{j} \leq B \\
0 \leq x_{j} \leq 1, j=1,2, \cdots, n,
\end{array}\right.
$$

in which the constraint guarantees that the expected value of the total cost required for the new/improved product will not exceed the expected value of the budget.

In some cases, an enterprise hopes to achieve a preferred acceptable overall customer satisfaction with the minimum possible design costs under the objective function, which can be described as

$$
\min E(C)=C_{F}+\sum_{j=1}^{n} E\left(c_{j}\right) x_{j} .
$$

Since $C_{F}$ is a constant, Formula (35) can be rewritten as

$$
\min E\left(C_{V}\right)=\sum_{j=1}^{n} E\left(c_{j}\right) x_{j}=\sum_{j=1}^{n}\left(\int_{0}^{1} \varphi_{j}^{-1}(\alpha) \mathrm{d} \alpha\right) x_{j} .
$$

Hence, a second uncertain programming model (UP-2) can be expressed as

$$
\left\{\begin{array}{l}
\min E\left(C_{V}\right)=\sum_{j=1}^{n} E\left(c_{j}\right) x_{j} \\
\text { subject to: } \\
\sum_{j=1}^{n} \bar{E}\left(v_{j}\right) x_{j} \geq S^{\prime} \\
0 \leq x_{j} \leq 1, j=1,2, \cdots, n,
\end{array}\right.
$$

where $S^{\prime}$ represents the preferred acceptable overall customer satisfaction. The definition of $S^{\prime}$ depends on the decision-makers' preference and subjectivity on customer satisfaction of products. 
Table 1 The house of quality of a motor car

\begin{tabular}{|c|c|c|c|c|c|c|}
\hline & Engineering characteristics & $\begin{array}{l}- \\
\mathrm{EC}_{1} \\
x_{1}\end{array}$ & $\begin{array}{l}+ \\
\mathrm{EC}_{2} \\
x_{2}\end{array}$ & $\begin{array}{l}- \\
\mathrm{EC}_{3} \\
x_{3}\end{array}$ & $\begin{array}{l}+ \\
\mathrm{EC}_{4} \\
x_{4}\end{array}$ & $\begin{array}{l}+ \\
E C_{5} \\
x_{5}\end{array}$ \\
\hline \multirow{5}{*}{ Uncertain correlation matrix } & $E C_{1}$ & $x^{6} \quad\left(\alpha^{1 / 6}\right)$ & $x^{1 / 9} \quad\left(\alpha^{9}\right)$ & $x^{1 / 9} \quad\left(\alpha^{9}\right)$ & $x^{1 / 9} \quad\left(\alpha^{9}\right)$ & $x^{1 / 9} \quad\left(\alpha^{9}\right)$ \\
\hline & $\mathrm{EC}_{2}$ & $x^{1 / 9} \quad\left(\alpha^{9}\right)$ & $x^{6} \quad\left(\alpha^{1 / 6}\right)$ & $x^{2} \quad\left(\alpha^{1 / 2}\right)$ & $x^{1 / 9} \quad\left(\alpha^{9}\right)$ & $x^{1 / 9} \quad\left(\alpha^{9}\right)$ \\
\hline & $\mathrm{EC}_{3}$ & $x^{1 / 9} \quad\left(\alpha^{9}\right)$ & $x^{2} \quad\left(\alpha^{1 / 2}\right)$ & $x^{6} \quad\left(\alpha^{1 / 6}\right)$ & $x^{4} \quad\left(\alpha^{1 / 4}\right)$ & $x^{1 / 9} \quad\left(\alpha^{9}\right)$ \\
\hline & $\mathrm{EC}_{4}$ & $x^{1 / 9} \quad\left(\alpha^{9}\right)$ & $x^{1 / 9} \quad\left(\alpha^{9}\right)$ & $x^{4} \quad\left(\alpha^{1 / 4}\right)$ & $x^{6} \quad\left(\alpha^{1 / 6}\right)$ & $x^{1 / 9} \quad\left(\alpha^{9}\right)$ \\
\hline & $\mathrm{EC}_{5}$ & $x^{1 / 9} \quad\left(\alpha^{9}\right)$ & $x^{1 / 9} \quad\left(\alpha^{9}\right)$ & $x^{1 / 9} \quad\left(\alpha^{9}\right)$ & $x^{1 / 9} \quad\left(\alpha^{9}\right)$ & $x^{6} \quad\left(\alpha^{1 / 6}\right)$ \\
\hline $\begin{array}{l}\text { Customer } \\
\text { requirements }\end{array}$ & $\begin{array}{l}\text { Uncertain weighs of } \\
\text { customer requirements }\end{array}$ & \multicolumn{5}{|c|}{ Uncertain relationship matrix between customer requirements and engineering characteristics } \\
\hline $\mathrm{CR}_{1} \quad y_{1}$ & $x^{4} \quad\left(\alpha^{1 / 4}\right)$ & $x^{6} \quad\left(\alpha^{1 / 6}\right)$ & $x^{1 / 9}\left(\alpha^{9}\right)$ & $x^{2}\left(\alpha^{1 / 2}\right)$ & $x^{1 / 9} \quad\left(\alpha^{9}\right)$ & $x^{1 / 9}\left(\alpha^{9}\right)$ \\
\hline $\mathrm{CR}_{2} \quad y_{2}$ & $x^{2} \quad\left(\alpha^{1 / 2}\right)$ & $x^{1 / 9} \quad\left(\alpha^{9}\right)$ & $x^{4} \quad\left(\alpha^{1 / 4}\right)$ & $x^{1 / 9} \quad\left(\alpha^{9}\right)$ & $x^{1 / 9} \quad\left(\alpha^{9}\right)$ & $x^{1 / 9} \quad\left(\alpha^{9}\right)$ \\
\hline $\mathrm{CR}_{3} \quad y_{3}$ & $x^{2} \quad\left(\alpha^{1 / 2}\right)$ & $x^{1 / 9} \quad\left(\alpha^{9}\right)$ & $x^{1 / 9} \quad\left(\alpha^{9}\right)$ & $x^{6} \quad\left(\alpha^{1 / 6}\right)$ & $x^{1 / 9} \quad\left(\alpha^{9}\right)$ & $x^{1 / 9} \quad\left(\alpha^{9}\right)$ \\
\hline $\mathrm{CR}_{4} \quad y_{4}$ & $x^{6} \quad\left(\alpha^{1 / 6}\right)$ & $x^{1 / 9} \quad\left(\alpha^{9}\right)$ & $x^{1 / 9} \quad\left(\alpha^{9}\right)$ & $x^{1 / 9} \quad\left(\alpha^{9}\right)$ & $x^{6} \quad\left(\alpha^{1 / 6}\right)$ & $x^{1 / 9} \quad\left(\alpha^{9}\right)$ \\
\hline \multirow[t]{5}{*}{$C R_{5} \quad y_{5}$} & $x^{1 / 4} \quad\left(\alpha^{4}\right)$ & $x^{1 / 9} \quad\left(\alpha^{9}\right)$ & $x^{1 / 9} \quad\left(\alpha^{9}\right)$ & $x^{1 / 9} \quad\left(\alpha^{9}\right)$ & $x^{1 / 9} \quad\left(\alpha^{9}\right)$ & $x^{4} \quad\left(\alpha^{1 / 4}\right)$ \\
\hline & Units & $d B$ & Horsepower & Gallon & $\mathrm{Kg}$ & $M^{3}$ \\
\hline & Comp $_{1}$ & 80 & 75 & 0.042 & 23 & 0.18 \\
\hline & $\mathrm{Comp}_{2}$ & 65 & 70 & 0.034 & 24 & 0.20 \\
\hline & $\mathrm{Comp}_{3}$ & 65 & 80 & 0.028 & 23 & 0.18 \\
\hline \multirow[t]{5}{*}{ Technical measures } & $\mathrm{Comp}_{4}$ & 75 & 60 & 0.032 & 15 & 0.14 \\
\hline & $\mathrm{Comp}_{5}$ & 95 & 80 & 0.030 & 20 & 0.19 \\
\hline & Min & 60 & 60 & 0.027 & 15 & 0.14 \\
\hline & Max & 95 & 90 & 0.042 & 25 & 0.21 \\
\hline & Cost coefficients & $\mathcal{L}(8,12)$ & $\mathcal{L}(9,12)$ & $\mathcal{L}(24,26)$ & $\mathcal{L}(14,16)$ & $\mathcal{L}(7,10)$ \\
\hline
\end{tabular}


Table 2 Different types of uncertain variables with different meanings for grading

\begin{tabular}{lllll}
\hline Type & Uncertain variables & W & R & P \\
\hline I & $\Phi(x)=x^{1 / 9}$ & extremely unimportant & extremely weak & strong negative \\
II & $\Phi(x)=x^{1 / 6}$ & very unimportant & very weak & very negative \\
III & $\Phi(x)=x^{1 / 4}$ & quite unimportant & quite weak & quite negative \\
IV & $\Phi(x)=x^{1 / 2}$ & some important & weak & weak negative \\
V & $\Phi(x)=x$ & moderately important & medium & medium \\
VI & $\Phi(x)=x^{2}$ & important & strong & weak positive \\
VII & $\Phi(x)=x^{4}$ & quite important & quite strong & quite positive \\
VIII & $\Phi(x)=x^{6}$ & very important & very strong & very positive \\
IX & $\Phi(x)=x^{9}$ & extremely important & extremely strong & strong positive \\
\hline & & & &
\end{tabular}

\section{Numerical Example}

To demonstrate the feasibility and effectiveness of the proposed uncertain programming models, the development of a new type of motor car is introduced as an example in this section. Applying QFD into the process aims to investigate the influence of target values of ECs on the overall customer satisfaction and the total design cost, which will provide a dynamic routine to guide the design team to determine a new set of target values for ECs.

A corporation is improving a new model of motor car to enhance competitiveness and occupy larger market, thus a survey regarding an initial market among users was done. With respect to the survey data in the market and feedbacks from users, five major CRs are identified to be the most significant concerns of the customers. i.e., "reducing the noise of car" $\left(\mathrm{CR}_{1}\right)$, "enhancing the acceleration" $\left(\mathrm{CR}_{2}\right)$, "saving fuel" $\left(\mathrm{CR}_{3}\right)$, "improving security" $\left(\mathrm{CR}_{4}\right)$, and "seat comfort" $\left(\mathrm{CR}_{5}\right)$, respectively.

Based on the design team's experience and expert knowledge on car, the five crucial ECs are identified, which are "reducing the noise of the exhaust system" $\left(\mathrm{EC}_{1}\right)$, "increasing the horsepower of the engine" $\left(E C_{2}\right)$, "reducing the amount of fuel per mile" $\left(E C_{3}\right)$, "increasing the controlling force of braking system" $\left(\mathrm{EC}_{4}\right)$, and "enlarging the space of

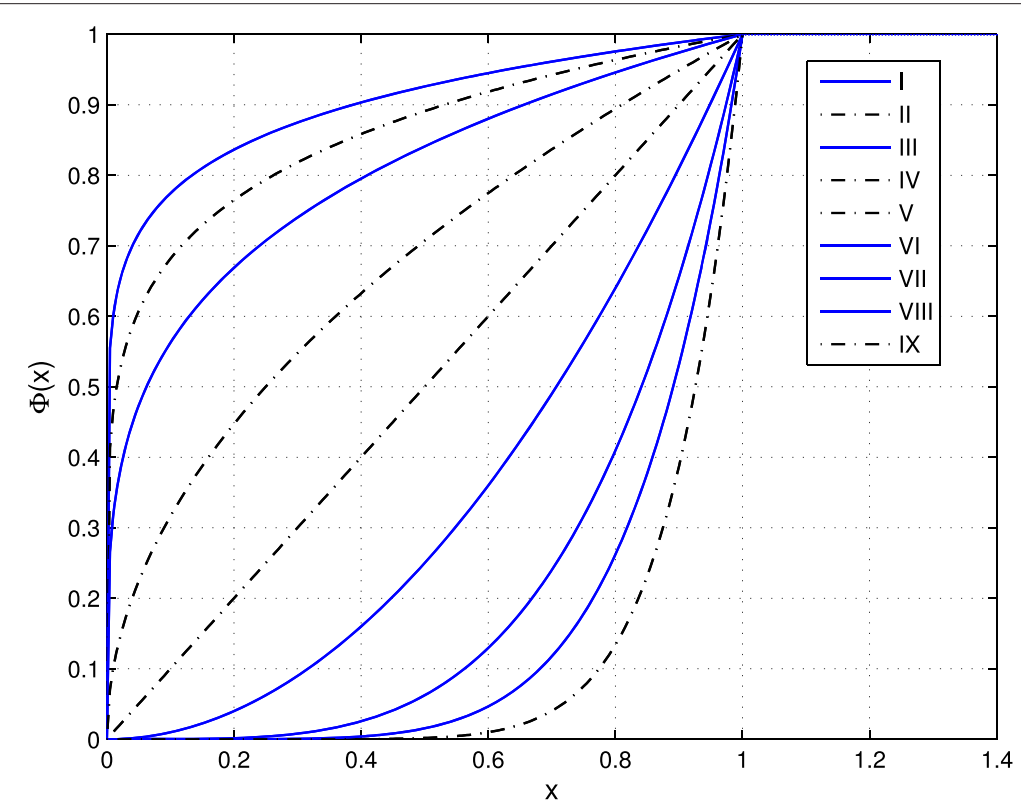

Fig. 5 Uncertain variables applied in HoQ of a motor car 
Table 3 Relative importance for five ECs through normalized expected values

\begin{tabular}{llllll}
\hline & $\mathrm{EC}_{1}$ & $\mathrm{EC}_{2}$ & $\mathrm{EC}_{3}$ & $\mathrm{EC}_{4}$ & $\mathrm{EC}_{5}$ \\
\hline$E\left(v_{j}\right)$ & 2.18 & 2.91 & 3.67 & 3.17 & 1.78 \\
$\bar{E}\left(v_{j}\right)$ & 0.16 & 0.21 & 0.27 & 0.23 & 0.13 \\
Ranking & 4 & 3 & 1 & 2 & 5 \\
\hline
\end{tabular}

the seat" $\left(E_{5}\right)$, respectively. These ECs are measured in units of $d B$, horsepower, gallon, $\mathrm{kg}$, and $\mathrm{m}^{3}$. The positive/negative sign on ECs indicates the design team's wishes to increase/decrease the target values of various ECs. There are five main competitors, i.e., Comp 1 (our corporation), Comp 2 , Comp 3 , Comp 4 , and Comp 5 to be considered. Detailed information may refer to $\mathrm{HoQ}$ of a motor car presented in Table 1.

Through the $\mathrm{HoQ}$, some more illustrative forms of uncertainty distribution and inverse uncertainty distribution of uncertain variables can be seen in matrices $W, R$, and $P$. With respect to Section 3.1, a certain kind of uncertain variables has been put forward to describe subjective judgements from experts, the specific meanings of which are shown in Table 2. Among them, five uncertain variables have been adopted by experts to define the strength of relationship measure and correlation measure in $\mathrm{HoQ}$, which are depicted as solid lines in Fig. 5 while others are dash-dotted lines. Thereby, as introduced, I, VI, VII, and VIII indicate different degrees of strength, such as extremely weak, strong, quite strong, and very strong in matrix $R$ and strong negative, weak positive, quite positive, and very positive in matrix $P$, respectively. When it comes to matrix $W$, III, VI, VII, and VIII represent "quite unimportant", "important", "quite important" and "very important" correspondingly.

Thus, according to Formulae (28) and (29) through calculating the expected value of importance $v_{j}$ of five ECs, the values and their normalized ones are summarized in Table 3, together with their ranking.

Furthermore, based on Formulae (9) and (10), the current target values of ECs of all competitors in Table 1 can be normalized as follows,

$$
X=\left[\begin{array}{ccccc}
0.43 & 0.50 & 0 & 0.80 & 0.57 \\
0.86 & 0.33 & 0.53 & 0.90 & 0.86 \\
0.86 & 0.67 & 0.93 & 0.80 & 0.57 \\
0.57 & 0 & 0.67 & 0 & 0 \\
0 & 0.83 & 0.80 & 0.50 & 0.71
\end{array}\right] .
$$

After we obtained all the data in matrices $W, R, P$, and $X$, according to Formula (30), the evaluation of each company's overall customer satisfaction can be easily obtained through the relative importance of individual EC and the matrix $X$, which is shown in Table 4. It is indicated that the existing design of Comp 1 currently has a low score of $E(S)(0.43)$ and ranks 4th, which means it is much less competitive, while Comp 3 achieves the highest customer satisfaction (0.79) among the five competitors. Therefore, there is a need for Comp ${ }_{1}$ to rationalize its existing design to improve its competitiveness.

Table 4 Rankings for five companies through normalized expected values

\begin{tabular}{llllll}
\hline & Comp $_{1}$ & Comp $_{2}$ & Comp $_{3}$ & Comp $_{4}$ & Comp $_{5}$ \\
\hline $\bar{E}\left(S_{q}\right)$ & 0.43 & 0.67 & 0.79 & 0.27 & 0.60 \\
Ranking & 4 & 2 & 1 & 5 & 3 \\
\hline
\end{tabular}


Table 5 Solutions of UP-1

\begin{tabular}{llllll}
\hline & $E C_{1}$ & $\mathrm{EC}_{2}$ & $\mathrm{EC}_{3}$ & $\mathrm{EC}_{4}$ & $\mathrm{EC}_{5}$ \\
\hline$x_{j}$ & 1 & 1 & 0.24 & 1 & 1 \\
$l_{j}$ & 60 & 90 & 0.0384 & 25 & 0.21 \\
$\bar{E}\left(v_{j}\right) / E\left(c_{j}\right)$ & 0.0160 & 0.0200 & 0.0108 & 0.0153 & 0.0152 \\
Ranking & 2 & 1 & 5 & 3 & 4 \\
\hline
\end{tabular}

In order to improve the existing design process, the resources are needed to be allocated more properly under the limit of a budget which will cover equipments and materials. Through investigation, the budget is determined to be 100 units in terms if all the resources are taken into consideration. As illustrated in Section 3.4, $C_{F}$ is the fixed cost in the development of design process which will set to be 50 units in this numerical example while $C_{j}$ is the variable part required to improve one unit of $\mathrm{EC}_{j}$, and each $c_{j}$ of individual $\mathrm{EC}_{j}$ is uncertain to determine. Thus, as shown in Table 1, five linear uncertain variables are applied to $c_{j}$ to express such circumstance.

As illustrated above, the expected value of relative importance $v_{j}$ of individual $\mathrm{EC}_{j}$ has been calculated. Besides, the budget, fixed and variable cost of the design process have been settled. With respect to Formula (33), then UP-1 in (34) can be rewritten as

$$
\left\{\begin{array}{l}
\max \bar{E}(S)=0.16 x_{1}+0.21 x_{2}+0.27 x_{3}+0.23 x_{4}+0.13 x_{5} \\
\text { subject to: } \\
\quad 10 x_{1}+10.5 x_{2}+25 x_{3}+15 x_{4}+8.5 x_{5} \leq 50 \\
0 \leq x_{j} \leq 1, j=1,2, \cdots, n .
\end{array}\right.
$$

The solutions of UP-1 are represented in Table 5, which will achieve the overall customer satisfaction of 0.79 . The determined target values of ECs $l_{j}$ are obtained through the invertible functions of Formula (9). Furthermore, it is noticeable that the value of $x_{3}$ is the last to be optimized after $\mathrm{EC}_{1}, \mathrm{EC}_{2}, \mathrm{EC}_{4}$, and $\mathrm{EC}_{5}$ have been fulfilled. The reason lies in the third row in Table 5, by comparing the parameters $\bar{E}\left(v_{j}\right)$ and $E\left(c_{j}\right)$ in objective functions and constraints, if the value is higher than others, the unit investment of which will increase first, thus higher customer satisfaction will be gained, i.e., $\bar{E}\left(v_{3}\right) / E\left(c_{3}\right)$ gains the lowest value, which means last worth to be improved even though the importance of $\mathrm{EC}_{3}$ ranks foremost.

Meanwhile, with respect to different values of $S^{\prime}$ scaled in [0,1], the corresponding results for the second uncertain programming model UP-2 based on Formula (37) are shown in Table 6. When analyzing the results, it is observable that five ECs are improved

Table 6 Solutions of UP-2 with different values of $S^{\prime}$

\begin{tabular}{lllllll}
\hline$S^{\prime}$ & $x_{1}$ & $x_{2}$ & $x_{3}$ & $x_{4}$ & $x_{5}$ & $E\left(C_{V}\right)$ \\
\hline 0.1 & 0 & 0.4762 & 0 & 0 & 0 & 5.0001 \\
0.2 & 0 & 0.9524 & 0 & 0 & 0 & 10.0002 \\
0.3 & 0.5625 & 1 & 0 & 0 & 0 & 16.1250 \\
0.4 & 1 & 1 & 0 & 0.1304 & 0 & 22.4575 \\
0.5 & 1 & 1 & 0 & 0.5652 & 0 & 28.9795 \\
0.6 & 1 & 1 & 0 & 1 & 0 & 35.5000 \\
0.7 & 1 & 1 & 0 & 1 & 0.7693 & 42.0390 \\
0.8 & 1 & 1 & 0.2593 & 1 & 1 & 50.4825 \\
0.9 & 1 & 1 & 0.6296 & 1 & 1 & 59.7425 \\
1 & 1 & 1 & 1 & 1 & 1 & 69.0000 \\
\hline
\end{tabular}




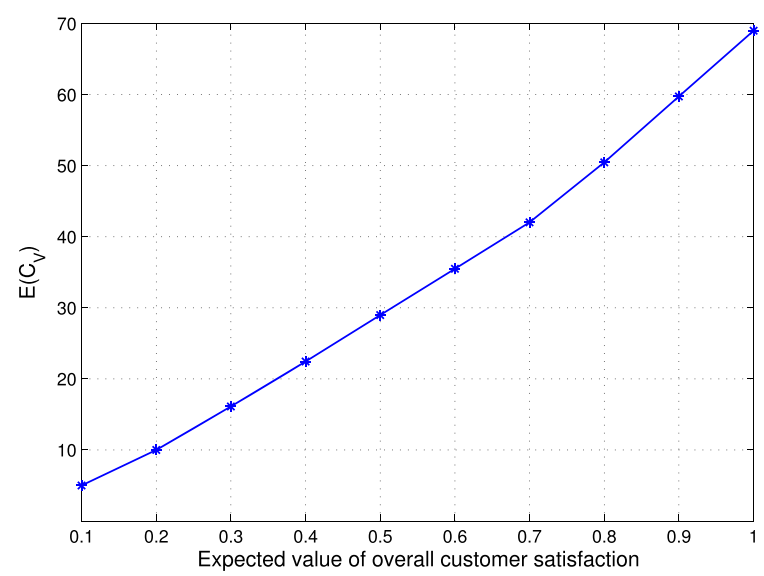

Fig. 6 Expected value of variable cost varies to different values of $S^{\prime}$

in the order of $\mathrm{EC}_{2}, \mathrm{EC}_{1}, \mathrm{EC}_{4}, \mathrm{EC}_{5}$, and $\mathrm{EC}_{3}$ as the value of $S^{\prime}$ increases. It can be explained according to the last row of Table 5 as well, the improved order is the same as the ranking of $\bar{E}\left(v_{j}\right) / E\left(c_{j}\right)$ in $\mathrm{EC}_{j}$. From Table 6, another conclusion can be drawn that the higher the value of $S^{\prime}$, the more the value of $E\left(C_{V}\right)$, the visualization of which is demonstrated in Fig. 6.

Through invertible functions of Formula (9), the target value of individual EC $l_{j}$ ( $j=$ $1,2, \cdots, n)$ for different value of $S^{\prime}$ can be obtained. In Formula (21), the variable cost $C_{j}$ required for improving each $\mathrm{EC}$ can be calculated by $c_{j} x_{j}$, respectively, $j=1,2, \cdots, n$, in which $c_{j}$ expressed as $\mathcal{L}\left(a_{j}, b_{j}\right)$. Furthermore, the lower limit and the upper limit of $C_{j}$ can be obtained, respectively, where $j=1,2, \cdots, n$. The relationships between $S^{\prime}$ and the target values of the five ECs and the corresponding variable costs incurred for improving them are demonstrated in Fig. 7a, b, Fig. 8a, b, Fig. 9a, b, Fig. 10a, b, and Fig. 11a, b, respectively.

The above five pairs of dynamic roadmaps would assist the design team to determine the target values of the five ECs to improve the design of motor car by taking competition requirements, the technical feasibility and financial factors into account. For example, if our company (Comp 1 ) wants to rank foremost among the competitors, the preferred acceptable overall customer satisfaction should at least match or exceed that of

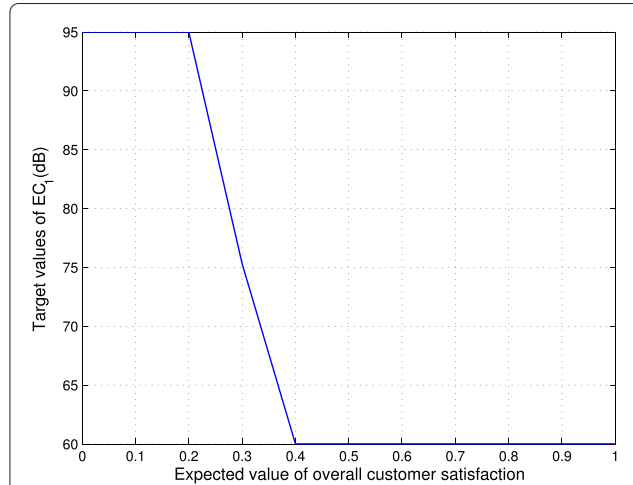

(a)

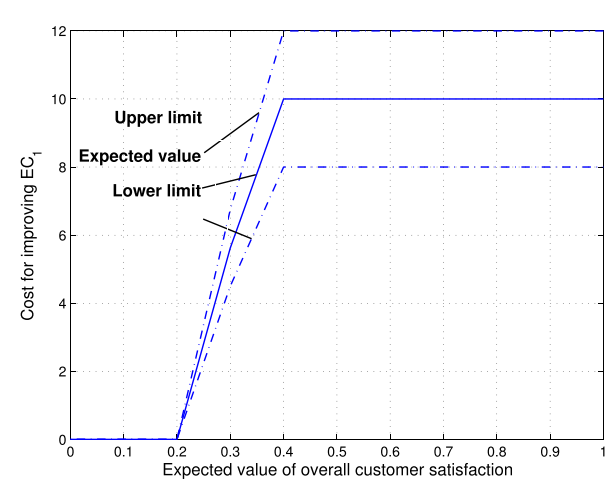

(b)

Fig. 7 a Relationship between $/_{1}$ and $S^{\prime}$. b Relationship between $C_{1}$ and $S^{\prime}$ 


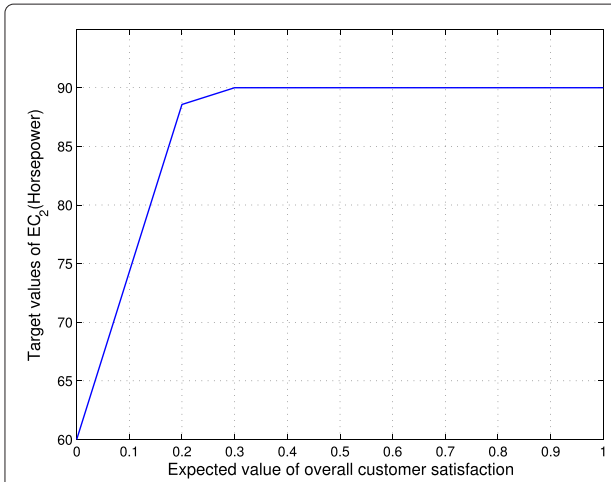

(a)

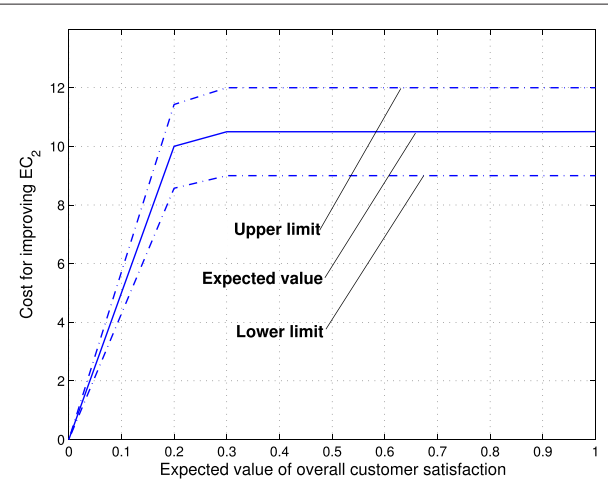

(b)

Fig. 8 a Relationship between $I_{2}$ and $S^{\prime}$. b Relationship between $C_{2}$ and $S^{\prime}$

Comp 3 (0.79), which is the current leader among five competitors. It will become more convenient for a design team to determine the target values for ECs of the improved car based on above five pairs of plots by uncertain programming, e.g., the calculated target values of ECs, $l_{1}$ is $60 \mathrm{~dB}, l_{2}$ is 90 horsepower, $l_{3}$ is smaller than 0.0384 gallon, $l_{4}$ is 25 $\mathrm{kg}$, and $l_{5}$ is $0.21 \mathrm{~m}^{3}$, respectively, along with the overall customer satisfaction of 0.79 . Accordingly, the variable costs for improving them are $(8,12),(9,12),(5.76,6.24),(14$, $16)$, and $(7,10)$. If the fixed cost is 50 units, the variable cost will be greater than $(43.76$, 56.24), which implies that the expected value of the total design cost will be at 100 units.

In resource constraints, the fixed cost and budget are crisp values pre-defined by experts; actually, it can also be defined as uncertain variables or other different crisp numbers in optimizing the models, which will lead to more comparative results.

To some extent, incorporating uncertainty theory into QFD would assist the company to better define the vagueness and ambiguity in the design process and achieve more rational results in determining the target values of ECs and obtain higher overall customer satisfaction.

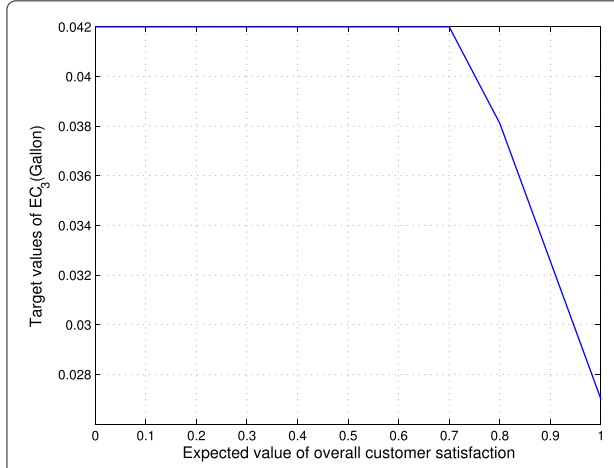

(a)

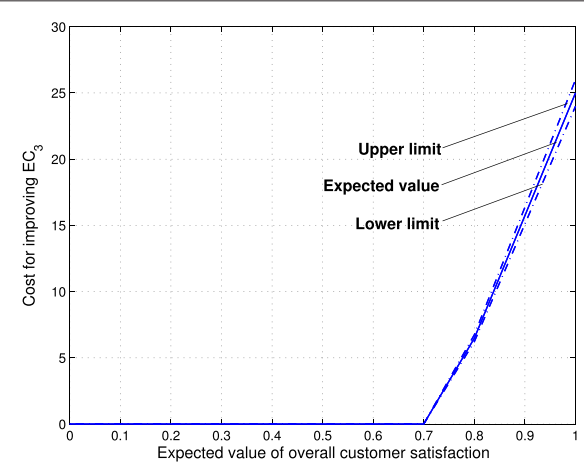

(b)

Fig. 9 a Relationship between $I_{3}$ and $S^{\prime}$. b Relationship between $C_{3}$ and $S^{\prime}$ 


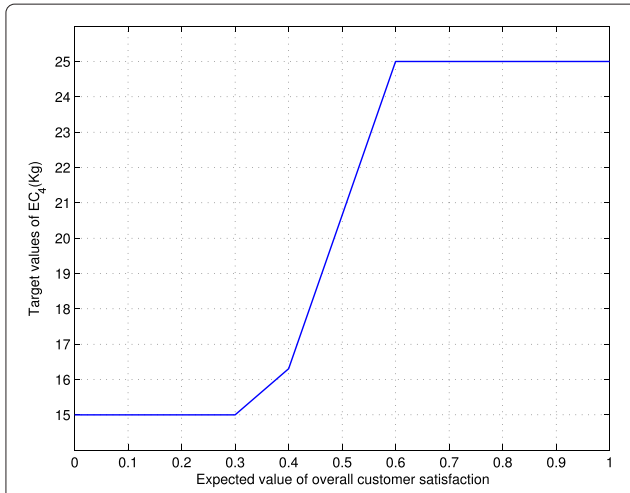

(a)

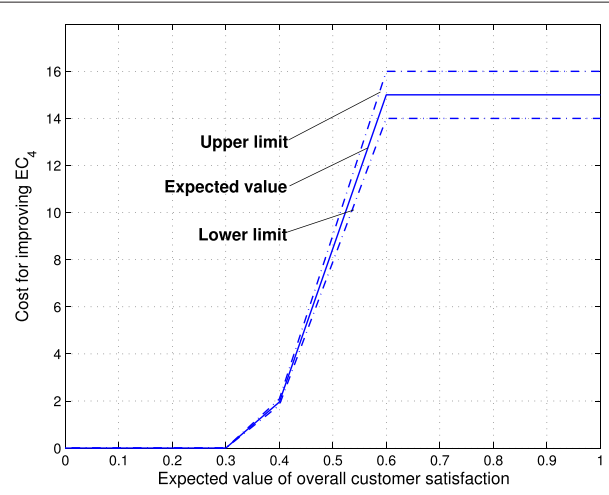

(b)

Fig. 10 a Relationship between $I_{4}$ and $S^{\prime}$. b Relationship between $C_{4}$ and $S^{\prime}$

\section{Conclusions}

In this paper, the basic idea of uncertain programming which includes expected value modeling has been applied to model the QFD planning process in an uncertain environment. On the basis of uncertainty theory, novel modeling approaches have been put up to determine the target values of ECs in QFD.

On account of the imprecise and uncertain elements in the development process, uncertain variables of regular uncertainty distributions have been adopted to define the relative importance of each CR, the uncertain relationship between CRs and ECs and the correlation among the ECs, while linear uncertain variables are applied to describe the variable cost of improving one unit of individual EC. The illustrated example of quality improved problem of a motor car showed that the proposed approach can model the process effectively in an uncertain environment by taking competition requirements, the technical feasibility and financial factors into consideration.

A new method based on uncertainty theory, namely, uncertain programming using EVM has been introduced in QFD to determine the target values of engineering characteristics in an uncertain environment of different real life scenarios. The work can be extended to many angles with respect to diverse uncertain features in HoQ. Much more can be done in this area, which may lead to more fruitful achievements.

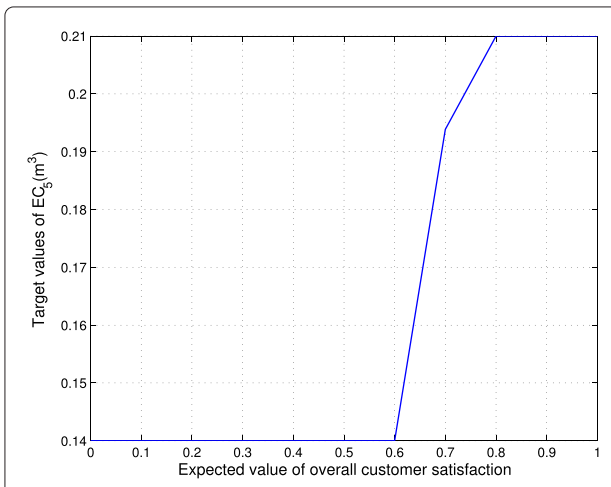

(a)

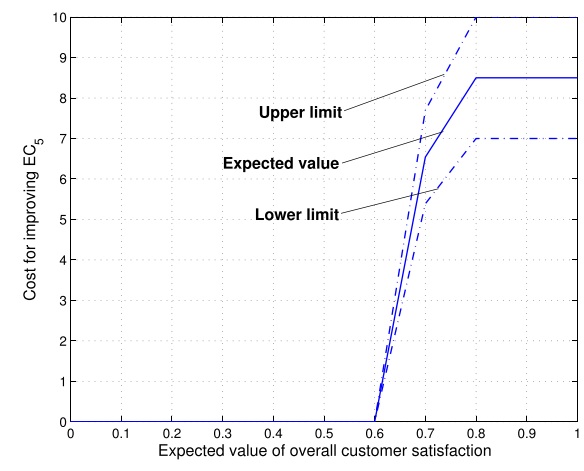

(b)

Fig. 11 a Relationship between $I_{5}$ and $S^{\prime}$. b Relationship between $C_{5}$ and $S^{\prime}$ 


\section{Competing interests}

The authors declare that they have no competing interests.

\section{Acknowledgements}

This work was supported in part by a grant from the National Natural Science Foundation of China (No. 71272177).

\section{Author details}

'School of Management, Shanghai University, 99 Shangda Road, 200444 Shanghai, China. ${ }^{2}$ Shenzhen Polytechnic, 518055 Shenzhen, China.

Received: 19 September 2015 Accepted: 10 November 2015

Published online: 08 December 2015

\section{References}

1. Akao, Y: Quality function deployment: Integrating customer requirements into product design. Productivity Press, Cambridge, Massachusetts (1990)

2. Katta, GDP, Kambagowni, VS, Kandukuri, NR: Supply chain design through QFD-based optimization. Int. J. Adv. Manuf. Tech. 25(5), 712-733 (2014)

3. Tidwell, A, Sutterfield, JS: Supplier selection using QFD: a consumer products case study. Int. J. Qual. Reliab. Manag. 29(3), 284-294 (2012)

4. Celik, M, Cebi, S, Kahraman, C, Er, D: An integrated fuzzy QFD model proposal on routing of shipping investment decisions in crude oil tanker market. Expert Syst. Appl. 36(3), 6227-6235 (2009)

5. Chen, CC, Zhang, Q: Applying quality function deployment techniques in lead production project selection and assignment. Adv. Mater. Res. 945-949, 2954-2959 (2014)

6. Hauser, JR, Clausing, D: The house of quality. Harvard Bus. Rev. 66(3), 63-73 (1988)

7. Chuang, PT: Combining the analytic hierarchy process and quality function deployment for a location decision from a requirement perspective. Int. J. Adv. Manuf. Tech. 18(11), 842-849 (2001)

8. Jae, HP, Kwang, MY, Kyong, SK: A quality function deployment methodology with signal and noise ratio for improvement of Wasserman's weights. Int. J. Adv. Manuf. Tech. 26(5-6), 631-637 (2005)

9. $\mathrm{Liu}, \mathrm{CH}, \mathrm{Wu}, \mathrm{HH}$ : A fuzzy group decision-making approach in quality function deployment. Qual. Quant. 42(4), 527-540 (2008)

10. Shen, $X X, T a n, K C, X i e, M:$ The implementation of quality function deployment based on linguistic data. J. Intell. Manuf. 12(1), 65-75 (2001)

11. Chen, Y, Chen, L: A non-linear possibilistic regression approach to model functional relationships in product planning. Int. J. Adv. Manuf. Tech. 28(11-12), 1175-1181 (2006)

12. Chen, Y, Tang, J, Fung, RYK, Ren, Z: Fuzzy regression-based mathematical programming model for quality function deployment. Int. J. Prod. Res. 42(5), 1009-1027 (2004)

13. Fung, RYK, Chen, $Y$, Tang, J: Estimating the functional relationships for quality function deployment under uncertainties. Fuzzy Set. Syst. 157(1), 98-120 (2006)

14. Kwong, CK, Chen, Y, Chan, KY, Luo, X: A generalized fuzzy least-squares regression approach to modelling relationships in QFD. J. Eng. Design. 21(5), 601-613 (2010)

15. Zadeh, LA: Fuzzy sets. Inf. Control. 8(3), 338-353 (1965)

16. Chen, Y, Fung, RYK, Tang, J: Fuzzy expected value modelling approach for determining target values of engineering characteristics in QFD. Int. J. Prod. Res. 43(17), 3583-3604 (2005)

17. Sener, Z, Karsak, EE: A desision model for setting target levels in quality function deployment using nonlinear programming-based fuzzy regression and optimization. Int. J. Adv. Manuf. Tech. 48(9-12), 1173-1184 (2010)

18. Sener, Z, Karsak, EE: A combined fuzzy linear regression and fuzzy multiple objective programming approach for setting target levels in quality function deployment. Expert Syst. Appl. 38(4), 3015-3022 (2011)

19. Zhong, S, Zhou, J, Chen, Y: Determination of target values of engineering characteristics in QFD using a fuzzy chance-constrained modelling approach. Neurocomputing. 142(1), 125-135 (2014)

20. Liu, B: Uncertainty theory. 4th ed. Springer-Verlag, Berlin (2015)

21. Liu, B: Uncertainty theory. 2nd ed. Springer-Verlag, Berlin (2007)

22. Liu, B: Uncertainty theory: a branch of mathematics for modeling human uncertainty. Springer-Verlag, Berlin (2010)

23. Liu, B: Some research problems in uncertainty theory. J. Uncertain Syst. 3(1), 3-10 (2009)

24. Liu, YH, Ha, MH: Expected value of function of uncertain variables. J. Uncertain Syst. 4(3), 181-186 (2010)

25. Liu, J, Zhong, S, Zhao, M: Expected value-based method to determine the importance of engineering characteristics in QFD with uncertainty theory. J. Uncertain Syst. 8(4), 271-284 (2014)

26. Tang, J, Fung, RYK, Xu, B: A new approach to quality function deployment planning with financial consideration. Comput. Oper. Res. 29(11), 1447-1463 (2002)

27. Zhou, M: Fuzzy logic and optimization models for implementing QFD. Comput. Ind. Eng. 35(1), 237-240 (1998)

28. Park, T, Kim, KJ: Determination of an optimal set of design requirements using house of quality. J. Oper. Manag. 16, 469-581 (1998)

29. Liu, B: Theory and practice of uncertain programming. 2nd ed. Springer-Verlag, Berlin (2009)

30. Peng, J, Zhang, B, Li, S: Towards uncertain network optimization (2015). doi:10.1186/s40467-014-0022-4

31. Zhang, Y, Liu, P, Yang, L, Gao, Y: A bi-objective model for uncertain multi-modal shortest path problems, Vol. 3 (2015). doi:10.1186/s40467-015-0032-x

32. Liu, B, Chen, $X$ : Uncertain multiobjective programming and uncertain goal programming. J. Uncertainty Anal. Appl. 3(10) (2015). doi:10.1186/s40467-015-0036-6 